\title{
MATURAÇÃO E MORFOMETRIA DOS FRUTOS DE Miconia albicans (Swartz) Triana (MELASTOMATACEAE) EM UM REMANESCENTE DE FLORESTA ESTACIONAL SEMIDECÍDUA MONTANA EM LAVRAS, MG ${ }^{1}$
}

\author{
Fábio de Almeida Vieira² e Dulcinéia de Carvalho ${ }^{3}$
}

\begin{abstract}
RESUMO - Os objetivos deste trabalho foram analisar a dinâmica da maturação dos frutos e avaliar quantitativamente algumas características físicas dos frutos de Miconia albicans (Swartz) Triana em um remanescente de Floresta Estacional Semidecídua Montana. A atividade, intensidade e sincronia de 20 indivíduos foram analisadas em relação aos eventos de frutificação, correlacionando-os com as variáveis climáticas. Analisou-se a morfometria (comprimento, largura e massa) de 130 frutos de 10 indivíduos. A intensidade da fenofase de frutos maduros nas plantas correlacionou-se significativamente com a precipitação média do período $\left(r_{S}=0,611 ; P<0,05\right)$. A espécie possui número elevado de pequenas sementes por fruto $(\bar{x}=28,05 \pm 1,45$ d.p. $)$. O coeficiente de correlação entre a massa fresca do fruto e a da polpa foi de $r_{S}=0,988(P<0,001)$, ou seja, a massa fresca do fruto é linearmente proporcional à quantidade de polpa. Esta contribui, em média, com $94 \%$ da massa fresca total do fruto, demonstrando a importância da espécie para os frugívoros. Os resultados indicaram o período de alta sincronia intrapopulacional das fenofases analisadas, o que pode ser útil na orientação da coleta de sementes visando à criação de bancos de germoplasma e produção de mudas para a recuperação de áreas degradadas.
\end{abstract}

Palavras-chave: Fenologia, frutificação e sincronia.

\section{MATURATION AND MORPHOMETRICS OF THE FRUITS OF Miconia albicans (Swartz) Triana (MELASTOMATACEAE) IN A REMNANT OF MONTANE SEASONAL SEMIDECIDUOUS FOREST IN LAVRAS, MG}

\begin{abstract}
The aim of this study was to examine the dynamics of fruit maturation and quantitatively assess some physical characteristics of the fruits of Miconia albicans (Swartz) Triana in a remnant of Montane Seasonal Semideciduous Forest. The activity and synchrony of 20 individuals were analyzed in regard to the proportion of fruiting events, and to help to determine their correlation to abiotic factors. Morphometric traits (fruit length, diameter and mass) of 130 fruits from ten individuals were analyzed. The number of fruits maturing showed a significant correlation with the mean precipitation $\left(r_{S}=0.611 ; P<0.05\right)$. M. albicans presented a high number of small seeds per fruit $(\bar{x}=28.05 \pm 1.45$ s.d. $)$. The fresh mass of the fruit was approximately equal to the pulp mass $\left(r_{S}=0.988 ; P<0.05\right)$. Thepulp contributed with $94 \%$ of the total mass, demonstrating the potential importance of this species for frugivores. The results indicate the period of high intrapopulation synchrony of the studied phenophases, which can be a useful guide in the collection of seeds for germoplasm banks and recovery of degraded areas.
\end{abstract}

Keywords: Phenology, fruiting and synchrony.

\section{INTRODUÇÃO}

O entendimento dos padrões fenológicos nos ecossistemas naturais é informativo para programas de conservação de recursos genéticos e manejo florestal.
Esta abordagem assume importância, por exemplo, para a taxonomia (SOLIVA e WIDMER, 1999) e para as associações com os fatores ambientais (WRIGHT et al., 1999; GOULART et al., 2005), genéticos (El-KASSABY, 1984; SOLIVA e WIDMER, 1999), com a fragmentação

\footnotetext{
${ }^{1}$ Recebido em 15.02.2008 e aceito para publicação em 23.06.2009.

${ }^{2}$ Departamento de Agropecuária da Universidade Federal do Rio Grande do Norte - Natal, RN. E-mail: <vieirafa@ufrnet.br>.

${ }_{3}^{3}$ Departamento de Ciências Florestais da Universidade Federal de Lavras - Lavras, MG. E-mail: <dulce@ufla.br>.
} 
florestal (FUCHS et al., 2003; HERRERÍAS-DIEGO et al., 2006) e também para avaliar as possíveis respostas das comunidades vegetais às mudanças climáticas (CHAPMAN et al., 2005). Muitos estudos reuniram informações fenológicas reprodutivas para as espécies (DAVIES e ASHTON, 1999; MARQUES et al., 2004), mas, em geral, não examinaram diferenças dentro de populações em micro escala temporal. Esses estudos são importantes para entender a dinâmica fenológica como estratégia de sobrevivência das populações e como os fatores abióticos influenciam os padrões fenológicos, principalmente nas espécies que apresentam eventos reprodutivos em pulsos ou em curto período de tempo (LARSON e BARRET, 1999; MEAGHER e DELPH, 2001; MICHALSKI e DURKA, 2007).

Eventos fenológicos reprodutivos sazonais e sincronizados podem representar vantagens adaptativas para muitas espécies tropicais (FRANKIE et al., 1974). Os eventos de frutificação são determinantes para o sucesso reprodutivo das populações e podem influenciar a atração de dispersores de sementes. A produção sincronizada de frutos pode saciar os predadores de sementes e aumentar a atratividade de frugívoros necessários à dispersão das sementes (JANZEN, 1971; AUGSPURGER, 1981). Portanto, os eventos de frutificação influenciam a estrutura, funcionamento e regeneração das comunidades vegetais (WILLIAMS et al., 1999). Nos trópicos, fatores climáticos estão frequentemente associados com a sazonalidade dos eventos fenológicos das plantas (CAMARGO-RICALDE et al., 2004; MORELLATO, 2004).

A caracterização morfométrica dos frutos e sementes tem importância para a taxonomia e identificação de variedades e para verificar a ocorrência de variações fenotípicas e nas associações com os fatores ambientais e genéticos (CARDOSO e LOMÔNACO, 2003; SILVA et al., 2007). Estudos nesse sentido são escassos e poderiam também dar suporte a programas de reflorestamento (VÁZQUEZ-YANES e ARÉCHIGA, 1996). As características morfométricas dos frutos, associadas aos eventos de frutificação, também estão relacionadas a características da dispersão e do estabelecimento de plântulas, permitindo inferir sobre qual modelo de coevolução planta-dispersor (plantas de baixo investimento ou plantas de alto investimento) as espécies vegetais se adequam (HOWE

R. Árvore, Viçosa-MG, v.33, n.6, p.1015-1023, 2009 e SMALLWOOD, 1982). Indivíduos de Miconia albicans produzem frutos utilizados por diversas espécies de aves frugívoras, em particular por aquelas oportunistas (SNOW, 1981). Como os frutos das melastomatáceas são ricos em carboidratos e possuem sementes pequenas (SNOW, 1981), os pássaros que se alimentam de invertebrados teriam condições de balancear sua alimentação ingerindo-os (SICK, 1997). Assim, a estratégia de produzir muitas sementes pequenas garantiria sua dispersão por pássaros oportunistas. Entretanto, inexistem as informações sobre a morfometria e caracterização física dos frutos de Miconia albicans, assim como sobre a dinâmica da frutificação e suas relações com fatores abióticos, o que poderia fornecer conhecimentos para a discussão da ecologia da reprodução da espécie.

Neste trabalho foi analisada a dinâmica da maturação dos frutos de Miconia albicans em uma população de borda em um remanescente florestal, por meio da atividade, intensidade e sincronia dos indivíduos em relação aos eventos fenológicos, a fim de verificar a época de ocorrência, duração dos eventos e a correlação com as variáveis climáticas, bem como caracterizar fisicamente os frutos da espécie.

\section{MATERIAL E MÉTODOS}

\subsection{Local de estudo}

O trabalho foi realizado em um fragmento florestal localizado no Campus da Universidade Federal de Lavras (UFLA), no Município de Lavras, Sul de Minas Gerais ( $21^{\circ} 13^{\prime} 17^{\prime \prime S}$ e $\left.44^{\circ} 57^{\prime} 47^{\prime \prime} \mathrm{W}\right)$, conhecido como Mata da Subestação, com área de 8,75 ha e altitudes variando entre 910 e $940 \mathrm{~m}$. O clima da região é do tipo Cwb de Köppen (mesotérmico com verões brandos e suaves e estiagens de invernos); a precipitação e a temperatura médias anuais são de 1.493 mm e 19,3 ${ }^{\circ} \mathrm{C}$, respectivamente, com $66 \%$ da precipitação ocorrendo no período de novembro a fevereiro. A vegetação classifica-se como Floresta Estacional Semidecídua Montana e insere-se no domínio da Mata Atlântica sensu lato (OLIVEIRA FILHO e FONTES, 2000). Nessa mata, o gênero Miconia está entre os de maior riqueza florística. Além disso, Miconia albicans é uma das espécies que se mostraram mais relacionadas com solos fortemente drenados, ácidos e pobres em nutrientes, em comparação com as demais espécies presentes na área (ESPÍRITO-SANTO et al., 2002). 


\subsection{Espécie do estudo}

O gênero Miconia Ruiz \& Pavon é o mais abundante da tribo Miconieae DC (Melastomataceae) e um dos maiores gêneros neotropicais, com aproximadamente 1.056 espécies conhecidas. A espécie Miconia albicans (Swartz) Triana é um arbusto, mas pode atingir porte arbóreo, que ocorre desde o Sul do México e Antilhas até o Paraguai e Paraná (GOLDENBERG, 2004). A espécie é característica de Cerrado, sendo comum em bordas de florestas (ESPÍRITO-SANTO et al., 2002; NERI et al., 2005). As folhas da espécie apresentam efeitos alelopáticos (GORLA e PEREZ, 1997) e antimicrobianos (CELOTTO et al., 2003). Os frutos são bagas de coloração rosada quando imaturos e verde-jade quando maduros. A espécie é capaz de produzir sementes por apomixia, sem polinização (GOLDENBERG e SHEPHERD, 1998).

\subsection{Frutificação}

Foram selecionadas, aleatoriamente, 20 plantas, Cujas alturas foram adotadas. O estudo foi realizado no período de setembro a dezembro de 2004, sendo o acompanhamento fenológico dos indivíduos realizado semanalmente durante todo esse período. Foram observadas as fenofases de frutificação (fruto imaturo e maduro) por meio do índice de atividade (porcentagem de indivíduos com a fenofase) e por notas de intensidade (porcentual de Fournier) em cada fase (BENCKE e MORELLATO, 2002; ANDREIS et al., 2005). As notas de intensidade atribuídas foram: 0 indicando a ausência da fenofase; e 1, 2, 3 e 4, a presença da fenofase, respectivamente nos intervalos de 1 a $25 \%, 26$ a $50 \%$, 51 a $75 \%$ e acima de $75 \%$ dos ramos apresentando a fenofase descrita. Foi calculada a sincronia intrapopulacional nos eventos de frutificação, sendo considerados períodos com alta sincronia aqueles que apresentavam mais que $60 \%$ da proporção de indivíduos com os eventos analisados, conforme Bencke e Morellato (2002). Os dados fenológicos foram correlacionados com registros meteorológicos de temperatura, precipitação, umidade relativa, insolação e evapotranspiração, provenientes da Estação Meteorológica da UFLA.

\subsection{Morfometria dos frutos}

Os frutos maduros de Miconia albicans foram coletados diretamente nas copas dos indivíduos, num total de 10 , em novembro de 2004, as matrizes localizadas por caminhadas abrangendo a área de estudo. Em seguida, os frutos foram colocados em sacos de polietileno e conduzidos ao Laboratório de Conservação Genética de Espécies Arbóreas do Departamento de Ciências Florestais da UFLA, onde foram avaliados. Selecionaramse os frutos dos 10 indivíduos, descartando aqueles visualmente danificados e separando-os em 13 repetições. Após esse procedimento, iniciaram-se as avaliações de comprimento e largura de 130 frutos, com o auxílio de um paquímetro digital. Após medir o tamanho e a massa de matéria fresca dos frutos, estes foram despolpados manualmente, para avaliar a massa fresca das sementes (obtida do total de sementes por fruto), assim como mensurar a massa fresca da polpa por fruto. O rendimento de polpa para o total da amostra foi determinado subtraindo-se a massa fresca da semente do fruto inteiro. O teste de germinação das sementes foi conduzido em BOD ajustada à temperatura de 25 oC e fotoperíodo de $12 \mathrm{~h}$, apenas com a intenção de verificar a viabilidade das sementes. Estas foram colocadas em placas de Petri sobre papel previamente umedecido com água destilada.

\subsection{Análise dos dados de morfometria dos frutos}

Os dados de morfometria dos frutos e sementes foram analisados por meio de distribuição de frequência e por estatísticas univariadas que compreenderam medidas de posição e de dispersão: média $(\overline{\mathrm{x}})$, valores mínimo e máximo, coeficientes de variação (CV), de assimetria (S) e de curtose (K). Os valores de referência adotados para o coeficiente de assimetria foram $\mathrm{S}$ $<0$, distribuição assimétrica à esquerda e $S>0$, distribuição assimétrica à direita. Quanto ao coeficiente de curtose, os valores de referência foram $\mathrm{K}>3$, distribuição mais "afilada" que a normal (leptocúrtica); e $\mathrm{K}<3$, distribuição mais achatada do que a normal (platicúrtica). Esses dois coeficientes foram analisados estatisticamente pelo teste Qui-quadrado $\left(\chi^{2}\right)$, visando às comparações entre as frequências observadas e as esperadas para uma distribuição normal. Para verificar as diferenças entre indivíduos, os resultados foram submetidos à análise de variância pelo teste de Kruskal-Wallis (H) e as médias, comparadas pelo teste de Mann-Whitney (ZAR, 1999). Calculou-se o coeficiente de correlação não paramétrico de Spearman $\left(r_{S}\right)$ bem como a probabilidade associada $(a ́=0,05)$ entre os dados fenológicos e as variáveis climáticas e entre as características morfométricas dos frutos. Correções de Bonferroni foram aplicadas para avaliar os valores significativos (RICE, 1989).

R. Árvore, Viçosa-MG, v.33, n.6, p.1015-1023, 2009 

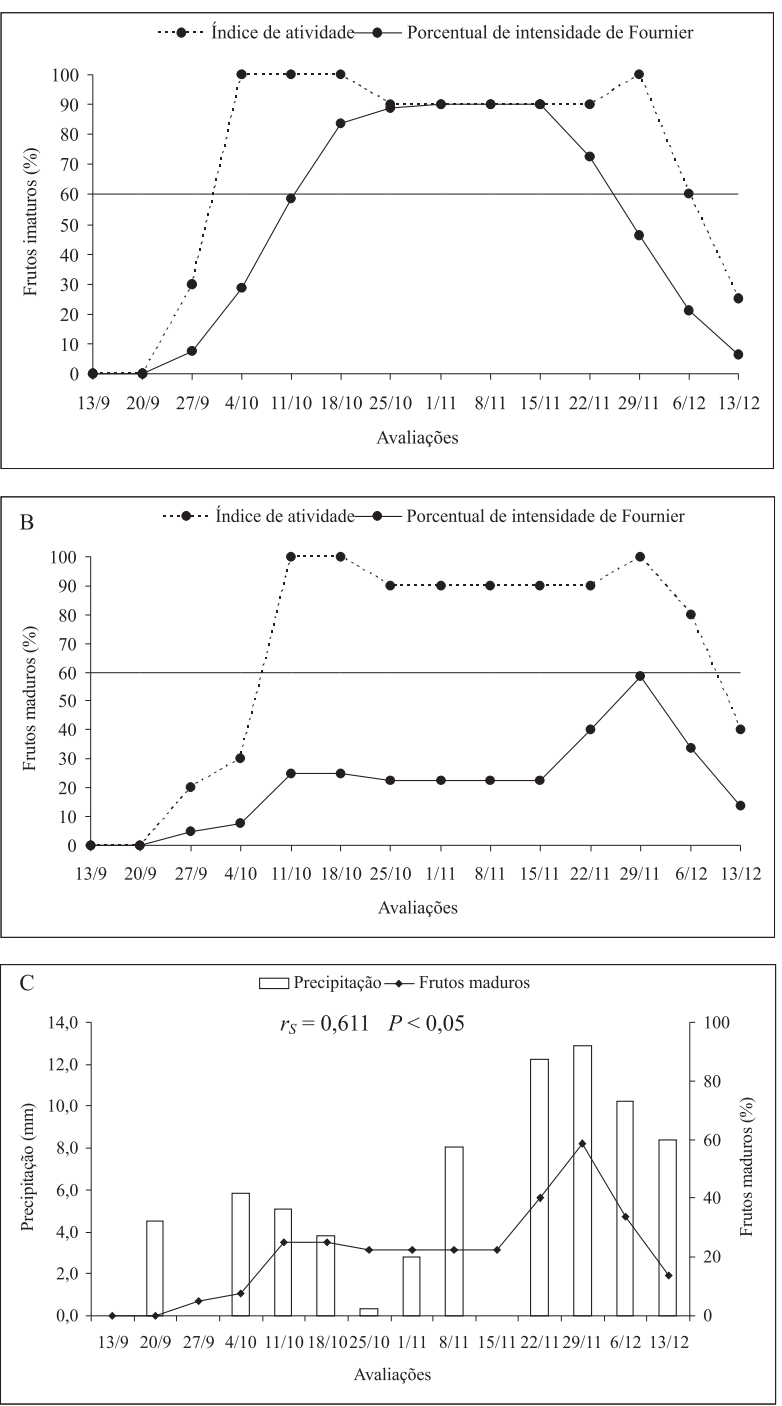

Figura 1 - Índices de atividade e percentual de intensidade de Fournier para as fenofases de frutos imaturos (A) e maduros (B). A linha na porcentagem 60 foi utilizada para destacar os períodos com alta sincronia (> 60\%) nos eventos da Figura AB. Correlação de Spearman $r_{s}(\mathrm{C})$ entre a precipitação e a intensidade de frutos maduros de Miconia albicans (Sw.) Triana.

Figure 1 - Activity indexes and percentage of intensity of Fournier for the phenophases of immature (A) and mature $(B)$ fruits. The line in the percentage 60 was used to detach the periods with high synchrony (> 60\%) in the events of the figure $A$ and $B$. Spearman's correlations $r_{S}(C)$ among the precipitation and the intensity of mature fruits of Miconia albicans (Sw.) Triana.

\section{RESULTADOS E DISCUSSÃO}

Os indivíduos de Miconia albicans na área de estudo são arbustos ou árvores encontrados em bordas do remanescente ou em clareiras. Os indivíduos apresentaram altura média de $\bar{x}=3,17 \mathrm{~m}$ (0,90 d.p.), cuja frutificação ocorreu tanto em indivíduos de pequeno porte $(0,7 \mathrm{~m}$ de altura) quanto em indivíduos maiores (5,2 $\mathrm{m}$ de altura). Frutos imaturos começaram a ser observados no mês de setembro (Figura 1A). O desenvolvimento dos frutos continuou até o início do mês de dezembro do mesmo ano. A sincronia intrapopulacional foi alta $(>60 \%)$ na fase de frutos imaturos no período compreendido entre o início do mês de outubro e fim de novembro. A intensidade máxima de frutos imaturos ocorreu entre a última semana de outubro e a primeira quinzena do mês de novembro. Frutos maduros foram observados até o final de dezembro, estação chuvosa (Figura 1B). Foi observada alta sincronia intrapopulacional no evento de frutos maduros no período entre $11 / 10$ e 6/12. A intensidade máxima da produção de frutos maduros ocorreu no final do mês de novembro. O desenvolvimento dos frutos é relativamente lento; o ovário começa a se dilatar e demora cerca de 20 a 30 dias para se desenvolver completamente. A intensidade de frutos maduros correlacionou-se significativa e positivamente com a precipitação média das normais climatológicas (Figura 1C). Assim, a estratégia de floração da espécie parece estar relacionada com o período ótimo de dispersão dos frutos e das sementes, pois o amadurecimento dos frutos foi maior na estação chuvosa. Geralmente, os frutos carnosos zoocóricos são produzidos durante a estação de maior precipitação (BATALHA e MANTOVANI, 2000). Todas as outras correlações entre os dados fenológicos e as variáveis climáticas não foram significativas.

A estimativa do período com maior sincronia intrapopulacional e intensidade de frutos maduros pode ser importante para a coleta de sementes visando à conservação ex situ e à exploração do germoplasma. Assim, o período entre 22/11 e 6/12 mostrou-se ideal para esse tipo de coleta, pois apresenta grande número de plantas com intensidade maior do evento. Esse período favorece, então, a amostragem aleatória das sementes nas matrizes, tomando-se o cuidado de amostrar igual número de sementes do maior número possível de plantas genitoras. Com essa prática, reduz-se a variância do número de gametas contribuídos pelas plantas genitoras, e aumenta-se o tamanho efetivo (VENCOVSKY, 1997). 
Tabela 1-Médias de massa de matéria fresca (MMF) de frutos, de polpas e sementes, comprimento (C) e diâmetro (D) dos frutos e número de sementes dos frutos de Miconia albicans (Sw.) Triana. ( $n=130$ frutos). e.p.: erro padrão; d.p.: desvio padrão; $C V$ : coeficiente de variação. $U$ : teste de Mann-Whitney; * $=P<0,05$ (após correções de Bonferroni); ns = não significativo.

Table 1 - Mean for fresh mass (MMF) of fruits, pulp and seeds, length $(C)$ and diameter $(D)$ of fruits and number of seeds of fruit of Miconia albicans $(S w$.$) Triana. (n=130$ fruits). e.p.: standard error; d.p.: standard deviation; CV: coefficient of variation. U: Mann-Whitney's test; $*=P<0.05$ (after Bonferroni corrections); $n s=$ not significant.

\begin{tabular}{|c|c|c|c|c|c|c|}
\hline Variáveis & $n$ & Média \pm e.p. & d.p. & $C V(\%)$ & $\operatorname{ANOVA}(H)$ & $U$ \\
\hline Fruto (MMF) (g) & 130 & $0,13 \pm 0,01$ & 0,02 & 14,7 & $13,22^{\text {ns }}$ & - \\
\hline Polpa (MMF) (g) & 130 & $0,13 \pm 0,00$ & 0,01 & 6,5 & $13,05^{\mathrm{ns}}$ & - \\
\hline Fruto $(\mathrm{C})(\mathrm{mm})$ & 130 & $4,90 \pm 0,06$ & 0,18 & 3,7 & $19,25 *$ & $U_{1 \times 6}=20 *$ \\
\hline Fruto (D) $(\mathrm{mm})$ & 130 & $6,62 \pm 0,16$ & 0,52 & 7,8 & $13,50^{\mathrm{ns}}$ & - \\
\hline Semente (MMF) (g) & 130 & $0,007 \pm 0,00$ & 0,00 & 11,3 & $12,47^{\mathrm{ns}}$ & - \\
\hline Número de sementes & 130 & $28,05 \pm 1,45$ & 4,58 & 16,3 & $7,87^{\text {ns }}$ & - \\
\hline
\end{tabular}

Isso pode ser importante no intuito de maximizar a diversidade genética populacional, principalmente considerando que a espécie é apomítica (GOLDENBERG e SHEPHERD, 1998). Adicionalmente, estudos em andamento sobre a estrutura genética em fina escala da espécie poderão subsidiar as questões sobre as estratégias de amostragem da população.

Os frutos de $M$. albicans são bagas globosas e de consistência suculenta. As sementes são numerosas, como na maioria das espécies da família Melastomataceae (GOLDENBERG, 2004), entre 12 e 40 por fruto, pequenas e com viabilidade de $100 \%$, com base na emissão da radícula. É possível que a abundância de sementes funcione como compensação, ajudando a sobrevivência da espécie, ao aumentar a chance de mais sementes encontrarem ambientes propícios com condições de luz, substrato e umidade necessários para o seu estabelecimento (MACARTHUR e WILSON, 1967).

Os dados morfométricos dos frutos de $M$. albicans indicaram que a amostragem foi adequada, uma vez que os valores do erro-padrão, com relação a todas as características avaliadas, foram pequenos, indicando que a média da amostra analisada está próxima da média da população, cujo valor é desconhecido (Tabela 1). O maior valor do desvio-padrão (d.p.) observado no número de sementes indica que houve maior variância amostral para essa característica biométrica, em relação às demais avaliadas. Quanto à variação entre indivíduos, em relação ao valor médio os valores do coeficiente de variação observados indicaram que a massa de matéria fresca do fruto e das sementes e o número de sementes variaram mais do que as outras características. Entretanto, apenas o comprimento do fruto apresentou variação significativa $(H=19,25$,
$\mathrm{P}<0,05)$, sendo verificada diferença entre as médias de duas plantas, conforme análise de variância ( $\left.\mathrm{U}_{\text {Planta } 1 \times 6}=20, \mathrm{P}<0,05\right)$.

O número de sementes dos frutos apresentou coeficiente de assimetria $(S)$ negativo (distribuição assimétrica à esquerda), evidenciando-se que frutos com maior número de sementes predominavam na amostra analisada (Figura 2). As demais características mostravam coeficientes de assimetria positivos (distribuição assimétrica à direita), indicando que frutos com menor massa total, comprimento e diâmetro e frutos com menor massa fresca da semente e de polpa predominavam na amostra analisada. Todas as variáveis biométricas apresentaram distribuição platicúrtica, conforme o coeficiente de curtose $(K<3)$. Isso aponta que a distribuição de frequência das variáveis analisadas é mais achatada do que a curva normal, ou seja, tem maior amplitude de distribuição dos dados. Apenas a variável diâmetro dos frutos não apresentou desvios significativos das frequências observadas em relação às frequências teóricas esperadas para uma distribuição normal, conforme teste do Qui-quadrado $\left(\chi^{2}\right)$.

O coeficiente de correlação entre a massa fresca do fruto e a da polpa foi de $r_{S}=0,988$ ( $\left.\mathrm{P}<0,001\right)$, ou seja, a massa fresca do fruto é linearmente proporcional à quantidade de polpa (Tabela 2). A polpa contribui, em média, com $94 \%$ da massa fresca total do fruto, o que demonstra alto rendimento, reafirmando a importância da espécie como fonte de alimento para a fauna. Outros estudos também costataram correlação positiva entre a massa de matéria fresca do fruto e a massa de matéria fresca da polpa (GUSMÃO et al., 2006; SILVA et al., 2007; VIEIRA e GUSMÃO, 2008). A ausência de correlações significativas entre as características morfométricas

R. Árvore, Viçosa-MG, v.33, n.6, p.1015-1023, 2009 

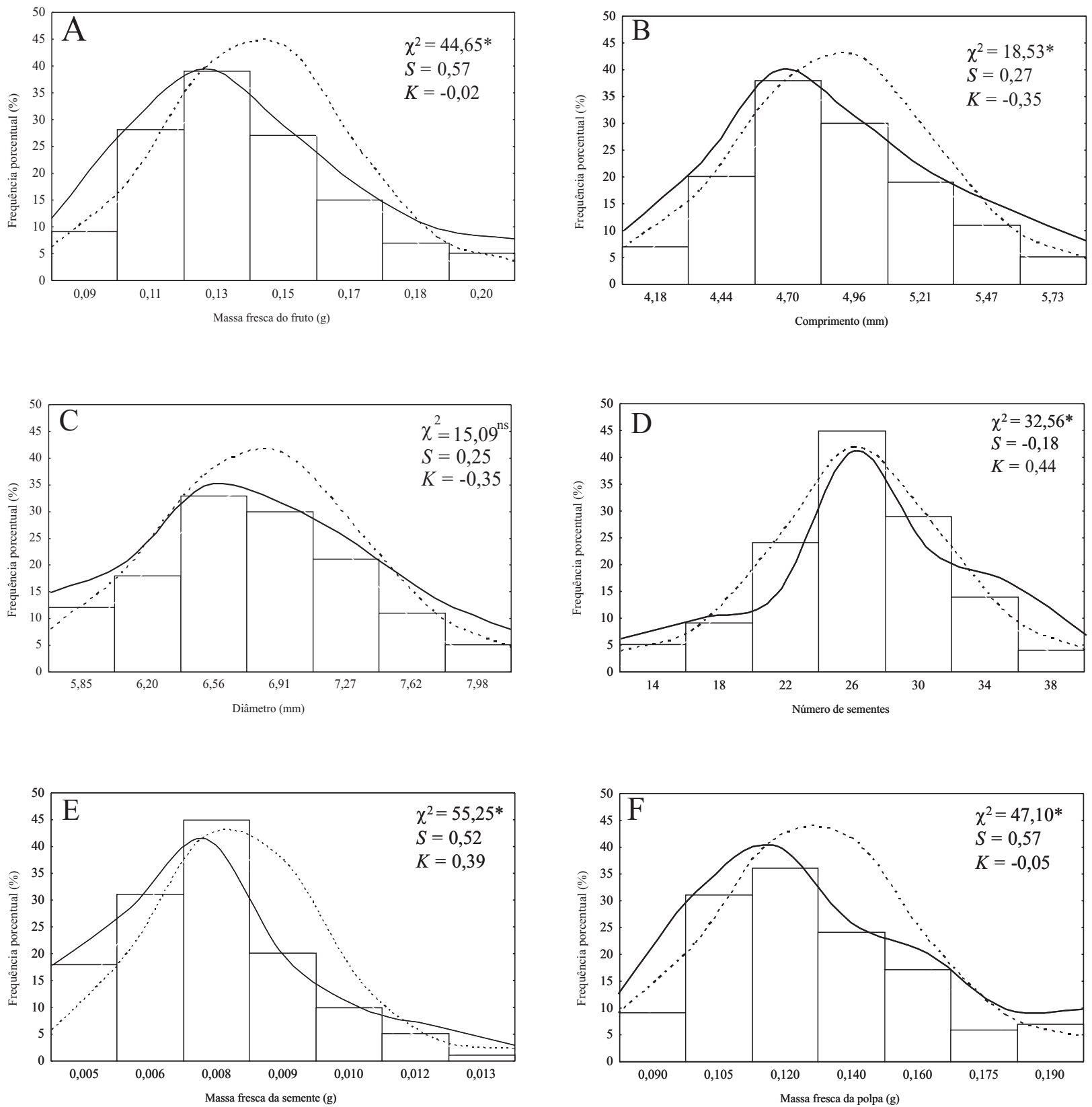

Figura 2 - Distribuição da frequência porcentual da massa de matéria fresca do fruto (A) comprimento (B) e diâmetro dos frutos (C), número de sementes (D), massa fresca das sementes (E) e massa fresca da polpa (F) dos frutos de Miconia albicans $(\mathrm{Sw}$.$) Triana. Barras e linhas contínuas =$ frequência observada; linhas pontilhadas = frequência esperada para uma distribuição normal; $\chi^{2}=$ teste de Qui-quadrado; $S=\operatorname{assimetria;} K=$ curtose; * significativo; e ns = não significativo (á $=0,05)$.

Figure 2 - Frequency percentuage distribution of the fresh mass of fruit $(A)$ length $(B)$ and diameter of fruits $(C)$, number of seeds $(D)$, fresh mass of seeds $(E)$ and fresh mass of the pulp $(F)$ of fruits of Miconia albicans (Sw.) Triana. Bar and continuous lines $=$ observed frequency; dotted lines $=$ expected frequency in a normal distribution. $\chi^{2}=$ Chi-square test $; S=$ skewness $; K=$ kurtosis $; *=$ significant, $n s=$ non significant $(a ́=0.05)$.

R. Árvore, Viçosa-MG, v.33, n.6, p.1015-1023, 2009 
Tabela 2 - Correlação de Spearman $\left(r_{s}\right)$ entre as variáveis morfométricas dos frutos de Miconia albicans (Sw.) Triana. * $=P<0,05$ (após correções de Bonferroni); $\mathrm{ns}=$ não significativo.

Table 2 - Spearman's correlations $\left(r_{s}\right)$ among morphometrics variables of fruits of Miconia albicans $($ Sw. $)$ Triana. * = $P<0.05$ (after Bonferroni corrections); ns = not significant.

\begin{tabular}{lr}
\hline \multicolumn{1}{c}{ Comparações } & $r_{S}$ \\
\hline Compr. do Fruto $x$ Diâm. do Fruto & $0,7333^{\mathrm{ns}}$ \\
Compr./Diâm. do Fruto $x$ Massa fresca do Fruto & $0,867 * / 0,855^{*}$ \\
Compr./Diâm. do Fruto $x$ Massa fresca da Polpa & $0,903 * / 0,879 *$ \\
Massa fresca do Fruto $x$ Massa fresca da Polpa & $0,988^{*}$ \\
Massa fresca do Fruto $x$ Número de Sementes & $0,588^{\mathrm{ns}}$ \\
Compr./Diâm. do Fruto $x$ Número de Sementes & $0,673^{\mathrm{ns}} / 0,370{ }^{\mathrm{ns}}$ \\
\hline
\end{tabular}

dos frutos e o número de sementes evidencia que o número de sementes provavelmente não é influenciado pelo tamanho do fruto.

Os resultados deste estudo indicaram o período de alta sincronia intrapopulacional das fenofases estudadas, que é importante na orientação da coleta de sementes visando à criação de bancos de germoplasma e para a produção de mudas para recuperação de áreas degradadas (MANTOVANI et al., 2003). Devido ao tamanho pequeno dos frutos maduros associado à alta produção de sementes $(\overline{\mathrm{x}}=28$ por fruto), à grande disponibilidade de frutos maduros e à provável maior frequência de ingestão destes por um frugívoro oportunista, considerase que $M$. albicans se encaixa no modelo de coevolução planta-dispersor do tipo "baixo investimento" (HOWE e SMALLWOOD, 1982). Entretanto, mais estudos são necessários para esclarecer as relações entre a espécie e seus agentes dispersores. Além disso, informações demográficas e da estrutura genética em fina escala poderão subsidiar as estratégias de amostragem e manejo da população. Assim, somente o acúmulo de informações pode apontar direções mais seguras para a compreensão da dinâmica da espécie e conservação das populações naturais.

\section{AGRADECIMENTOS}

À CAPES, pela bolsa de doutorado concedida ao primeiro autor; e ao CNPq, pela bolsa de Produtividade concedida ao segundo autor.

\section{REFERÊNCIAS}

ANDREIS, C. et al. Estudo fenológico em três fases sucessionais de uma floresta estacional decidual no município de Santa Tereza, RS, Brasil. Revista Árvore, v.29, n.1, p.55-63, 2005.
AUGSPURGER, C. K. Reproductive synchrony of a tropical plant: experimental effects of pollinators and seed predators on Hybanthus prunifolius (Violaceae). Ecology, v.62, n.3, p.755-788, 1981.

BATALHA, M. A.; MANTOVANI, W. Reproductive phenological patterns of cerrado plant species at the Pé-de-Gigante Reserve (Santa Rita do Passa Quatro, SP, Brazil): a comparison between the herbaceous and woody floras. Revista

Brasileira de Biologia, v.60, n.1, p.129-145, 2000.

BENCKE, C. S. C.; MORELLATO, L. P. C. Estudo comparativo da fenologia de nove espécies arbóreas em três tipos de floresta atlântica no sudeste do Brasil. Revista Brasileira de Botânica, v.25, n.2, p.237-248, 2002.

CAMARGO-RICALDE, S. L.; DHILLION, S. S.; GARCÍA-GARCÍA, V. Phenology, and seed production and germination of seven endemic Mimosa species (Fabaceae-Mimosoideae) of the Tehuacán-Cuicatlán Valley, Mexico. Journal of Arid Environments, v.58, n.4, p.423-437, 2004.

CARDOSO, G. L.; LOMÔNACO, C. Variações fenotípicas e potencial plástico de Eugenia calycina Cambess. (Myrtaceae) em uma área de transição cerrado-vereda. Revista Brasileira de Botânica, v.26, n.1, p.131-140, 2003.

CELOTTO, A. C. et al. Evaluation of the in vitro antimicrobial activity of crude extracts of three Miconia species. Brazilian Journal of Microbiology, v.34, n.4, p.339-340, 2003.

CHAPMAN, C. A. et al. A long-term evaluation of fruiting phenology: importance of climate change. Journal of Tropical Ecology, v.21, n.1, p.1$14,2005$.

R. Árvore, Viçosa-MG, v.33, n.6, p.1015-1023, 2009 
DAVIES, S. J.; ASHTON, P. S. Phenology and fecundity in 11 sympatric pioneer species of Macaranga (Euphorbiaceae) in Borneo.

American Journal of Botany, v.86, n.12, p.1786-1795, 1999.

EL-KASSABY, Y. A.; FASHLER, A. M. K.; SZIKLAI, O. Reproductive phenology and its impact on genetically improved seed production in a Douglas-fir seed orchard. Silvae Genetica, v.33, n.4/5, p.120-125, 1984.

ESPÍRITO-SANTO, F. D. B. et al. Variáveis ambientais e a distribuição de espécies arbóreas em um remanescente de floresta estacional semidecidual montana no campus da Universidade Federal de Lavras, MG. Acta Botanica Brasílica, v.16, n.3, p.331-356, 2002.

FRANKIE, G. W.; BAKER, H. G.; OPLER, P. A. A comparative phenological studies of trees in tropical wet and dry forest in the lowlands of Costa Rica. Journal of Ecology, v.62, n.3, p.881-919, 1974.

FUCHS, E. J.; LOBO, J. A.; QUESADA, M. Effects of forest fragmentation and flowering phenology on the reproductive success and mating patterns of the tropical dry forest tree Pachira quinata. Conservation Biology, v.17, n.1, p.149-157, 2003.

GOLDENBERG, R. O gênero Miconia

(Melastomataceae) no Estado do Paraná, Brasil. Acta Botanica Brasilica, v.18, n.4, p.927947, 2004.

GOLDENBERG, R.; SHEPHERD, G. J. Studies on the reproductive biology of Melastomataceae in "cerrado" vegetation. Plant Systematic Evolution, v.211, n.1, p.13-29, 1998.

GORLA, C. M.; PEREZ, S. C. J. G. A. Influência de extratos aquosos de folhas de Miconia albicans Triana, Lantana camara L., Leucaena leucocephala (Lam) de Wit e Drimys winteri Forst, na germinação e crescimento inicial de sementes de tomate e pepino. Revista

Brasileira de Sementes, v.19, n.2, p.260265, 1997.

R. Árvore, Viçosa-MG, v.33, n.6, p.1015-1023, 2009
GOULART, M. F.; LEMOS FILHO, J. P.; LOVATO, M. B. Phenological variation within and among populations of Plathymenia reticulate in Brazilian Cerrado, the Atlantic Forest and transitional sites. Annals of Botany, v.96, NUMERO, p.445-455, 2005.

GUSMÃO, E.; VIEIRA, F. A.; FONSECA JÚNIOR, E. M. Biometria de frutos e endocarpos de murici (Byrsonima verbascifolia Rich. ex A. Juss.). Cerne, v.12, n.1, p.84-91, 2006.

HERRERÍIAS-DIEGO, Y. et al. Effects of forest fragmentation on phenological patterns and reproductive success of the Tropical Dry Forest tree Ceiba aesculifolia. Conservation Biology, v.20, n.4, p.1111-1120, 2006.

HOWE, H. F.; SMALLWOOD, J. Ecology of seed dispersal. Annual Review of Ecology and Systematic, v.13, p.201-228, 1982.

JANZEN, D. H. Seed predation by animals. Annual Review of Ecology and Systematics, v.2, p.465-492, 1971.

LARSON, B. M. H.; BARRET, S. C. H. The ecology of pollen limitation in buzz-pollinated Rhexia virginica (Melastomataceae). Journal of Ecology, v.87, p.371-381, 1999.

MACARTHUR, R. H.; WILSON, E. O. The theory of island biogeography. Princeton: Princeton University Press, 1967. 203p.

MANTOVANI, M. et al. Fenologia reprodutiva de espécies arbóreas em uma formação secundária da Floresta Atlântica, Revista Árvore, v.27, n.4, p.451-458, 2003.

MARQUES, M. C. M.; ROPER, J. J.; SALVALAGGIO, A. P. B. Phenological patterns among plant life forms in a Subtropical Forest in Southern Brazil. Plant Ecology, v.173, p.203-213, 2004.

MEAGHER,T. R.; DELPH, L. F. Individual flower demography, floral phenology and floral display size in Silene latifolia. Evolutionary Ecology Research, v.3, p.845-860, 2001. 
MICHALSKI, S. G.; DURKA, W. Synchronous pulsed flowering: analysis of the flowering phenology in Juncus (Juncaceae) Annals of Botany, v.100, p.1271-1285, 2007.

MORELLATO, L. P. C. Phenology, sex ratio, and spatial distribution among dioecious species of Trichilia (Meliaceae). Plant Biology, v.6, p.491-497, 2004.

NERI, A. V. et al. Regeneração de espécies nativas lenhosas sob plantio de Eucalyptus em área de Cerrado na Floresta Nacional de Paraopeba, MG, Brasil. Acta Botanica Brasilica, v.19, n.2, p.369-376, 2005.

OLIVEIRA FILHO, A. T.; FONTES, M. A. L. Patterns of floristic differentiation among Atlantic forests in south-eastern Brazil and the influence of climate. Biotropica, v.32, p.793-810, 2000.

RICE, W. R. Analyzing tables of statistical tests. Evolution, v.43, p.223-225, 1989.

SICK, H. Ornitologia brasileira. Rio de Janeiro, Nova Fronteira, 1997. 861p.

SILVA, M. S.; VIEIRA, F. A.; CARVALHO, D. Biometria dos frutos e divergência genética em uma população de Geonoma schottiana Mart. Revista Brasileira de Biociências, v.5, S1, p.582-584, 2007.

SNOW, D. W. Tropical frugivorous birds and their food plants: a world survey. Biotropica, v.13, n.1, p.1-14, 1981.
SOLIVA, M.; WIDMER, A. Genetic and floral divergence among sympatric populations of Gymnadenia conopsea S.L. (Orchideaceae) with different flowering phenology. International Journal of Plant Science, v.160, n.5, p.897905, 1999.

VÁZQUEZ-YANES, C.; ARÉCHIGA, M. R. Ex situ conservation of tropical rain forest seed: problems and perspectives. Interciencia, v.21, p.293-298, 1996.

VENCOVSKY, R. Biometrical approaches for molecular markes: estimation of effective population size. In: INTERNATIONAL WORKSHOPON AGRICULTURALBIOTECHNOLOGY, 1997, Pircicaba. Proceedings...Piracicaba: ESALQ-USP; Cook College: New Jersey Agricultural Experiment Station, The State University of New Jersey, 1997. $2 \mathrm{p}$.

VIEIRA, F. A.; GUSMÃO, E. Biometria, armazenamento de sementes e emergência de plântulas de Talisia esculenta Radlk. (Sapindaceae). Ciência e Agrotecnologia, v.32, n.4, p.10731079, 2008.

WILLIAMS, R. J. et al. Reproductive phenology of woody species in a North Australian Tropical savanna. Biotropica, v.31, p.626-636, 1999.

WRIGHT, S. J. et al. The El Niño Southern Oscillation, variable fruit production, and famine in a tropical forest. Ecology, v.80, n.5, p.1632-1647, 1999.

ZAR, J. H. Biostatistical analysis. New Jersey: Prentice-Hall, 1999.663p. 
